\title{
Systems of vibration parameters automated control for diagnostics of equipment technical state
}

\author{
Serhiy Horiashchenko ${ }^{1,}{ }^{*}$, Oleg Polishchuk ${ }^{1}$, Marcin Łukasiewicz ${ }^{2}$, Maciej M atuszewski ${ }^{2}$ \\ and Vladimir Boykov ${ }^{3}$ \\ ${ }^{1}$ K hmel nitsky N ational U niversity, Instytutska Street 11, K hmel nytskyi, 29000, U kraine \\ 2UTP University of Science and Technology, Faculty of Mechanical Engineering, Al. prof. S. \\ Kaliskiego 7, Bydgoszcz, 85-796, Poland \\ ${ }^{3}$ B elarusian N ational Technical U niversity, 220013 M insk, N ezavisimosti, 65, B elarus
}

\begin{abstract}
The article deals with the issues of the spatial direction of measurement, which is optimal in terms of recognition of the state of a particular unit node and preparation of control points for diagnosis. To form a diagnostic feature, the representation of the signal in a rather narrow range has been used, for instance, in the area of one of the harmonics of the fundamental mechanism excitation frequency. To process such signals, an integrated programming environment developed in the style of Microsoft $V$ isual products has been created. To ensure the requirements of optimal vibration analysis, the structure of stationary equipment control systems has been proposed. A nalysis of random vibration of the diagnosed object is done using real-time two-channel analyzers. Each analyzer channel has a processor for fast Fourier transform and operational information. The two available channels can allow assessment of the state of the object by special correlation functions. In contrast to the known methods, the established methods of determining the frequency range of defects of various light industry machine nodes differ in taking account size of parts and equipment nodes, and the possibility to use narrow-band filtering. The analysis of possibilities to install vibration sensors has been carried out.
\end{abstract}

\section{Introduction}

When diagnosing equipment and assessing performance of each unit, there is always the issue of spatial direction of measurements, which is optimal in terms of recognition of the state of a particular unit node and preparation of control points for diagnosis. There is no complete answer to this question, but if it is impossible to carry out measurements in three main directions in the area of one bearing, or one needs to minimize the number of measurements, it is permissible to measure vibration in two directions: axial and one of the transverse directions. Preference is given to the transverse direction, usually corresponding to the direction of the system's minimum rigidity. It is also permissible to measure the axial vibration of the drive, supercharger and other unit nodes only at the bearing of the free end of the shaft.

Since the reactions of mechanical systems to excitation by mechanical oscillations are determined by complex physical processes, when measuring even at one element of the unit at points of introduction, close to each other, the different nature of the studied oscillations can be observed. The above-mentioned is especially relevant for the high-frequency component of the vibration signal, which is often determined by different types of distribution of high-frequency oscillations on the surface. It is important to measure vibration in the same spots, called control (regular) vibration measurement points [1].

* Corresponding author: gsl7@ukr.net 


\section{Problem statement}

It is known that the response of a mechanical system to the action of a short-term single pulse with a broadband spectrum can be observed at natural frequencies of the system. But the response energy at each of these frequencies was small even when acting on the impulse sequence system, which is why data of oscillations spectrum analysis at defects origination, such as chipping, cracking, and tearing do not allow to determine the type of defect with sufficient reliability. The power spectrum, being the average energy characteristic of the signal, is suitable only for the recognition of pre-emergency situations that are on the verge of crucial changes in the mechanism. To detect defects at an early stage, it is necessary to attract more informative characteristics, such as the number of signal emissions per time unit, exceeding a certain threshold value, the depth of amplitude and phase modulation, envel ope spectrum, cepstrum [2].

M ost methods of defects diagnostics in originating mechanisms are based on the same starting diagnostic model: development of the defect causes an increase in the amplitudes and number of short-term pulses in the vibration signal. The task is to apply the optimal method of signal processing, which minimizes the impact of noise and allows to unambiguously correlate the obtained signal characteristics with the type of defect [3]. The nature of the change in the structure of the vibration signal at fault detection determines the method of its processing to highlight the informative component that characterizes the change in the technical condition of the object diagnosed. Depending on the type of the unit and the type of defect that has arisen, different ways to increase the signal-to-noise ratio are used.

If the physics of fault impact on the oscillations of the mechanism is associated with the appearance of amplitude or phase modulation, the properties of the envelope vibration signal are researched.

Appearance in the signal or amplification of the periodic component calls for an effective way to select the periodic signal against noise background, for instance, to the method of synchronous accumulation.

A ppearance or amplification of polyharmonic oscillations can be detected by cepstral analysis, which compresses information about changes in the signal to a significant number of harmonics, the amplitudes of which are easy to quantify.

Occurrence and development of defects in mechanisms lead to emergence of nonlinear effects, which are used for formation of diagnostic signs to receive more valuable diagnostic information than the one obtained on the basis of consideration of linear dynamic models. For this purpose, values of the amplitudes of combinational frequencies, depth of the amplitude modulation or frequency deviation are used.

The above-mentioned methods of analysis of vibro-acoustic processes allow to form peculiar diagnostic signs of the arising defects, even in those cases when the spectrum of power of a signal practically does not change. In the presence of invariance of diagnostic signs, the reliability of diagnosis is guaranteed even at an early stage of defect development [3].

Thus, the algorithm for analysis of correlation-spectral characteristics of the vibration signal includes: sampling of the vibration signal, digital filtering, calculation of informative parameters, determination of the technical condition of the object.

The sum of harmonic components, multiples of the fundamental excitation frequency, i.e. in the form of polyharmonic vibration, is determined by the formula:

$$
\mathrm{x}(\mathrm{t})=\sum \mathrm{a}_{\kappa} ¥ \cos \left(\mathrm{k} ¥ \omega_{r} \mathrm{t}+\phi_{\kappa}\right)
$$

In rotor units, one of the main frequencies of vibration excitation is the speed, which is hereinafter referred to as the rotor speed: 


$$
f_{r}=\omega_{\mathrm{r}} / 2 \pi,
$$

in which $\omega_{\mathrm{r}}$ - angular rotor speed.

In steady-state modes, the oscillation spectrum is discrete with spectral components at the rotor speed and its harmonics. $\left(k f_{r}\right)$.

Thus, the informative parameters in this model of oscillations are the values of discrete components amplitudes of the spectrum at the rotor speed and its harmonics and the rate of their change with increasing mechanism's operating time. The amplitudes of oscillations at the rotor frequencies are often determined by the magnitude of the imbalance, mismatch of the shafts, kinematic errors and the ratio of critical rotor speed to the working one.

In addition to the above-mentioned vibration, a multiple of the rotor speed, in the spectrum of the vibration signal of the rotor unit may be characterized by the following frequency components:

$$
f_{z}=k\left(f_{r} z\right), k=1,2,3 \ldots n,
$$

in which $z$ - the number of elements correlation at rotor shaft.

For gearing, $z$ is equal to the number of gear teeth; for turbine, pump and fan - to the number of blades on the impeller etc.

The model of polyharmonic excitation of oscillations in rotor units is a convenient form of representation of the oscillatory process spectrum, which allows to focus only on certain frequencies $k f_{r}$, multiple of the basic frequency of oscillations excitation $f_{r}$ of the unit being diagnosed. The primary process of localization of increased vibroactivity sources of the unit design is precisely to identify potential sources of excitation that cause oscillations at a given frequency.

Practical experience with vibrodiagnostics of rotor units has shown that the polyharmonic model of vibration signal is only a zero approximation in the description of complex oscillatory process of a real unit, although it can be successfully used in source localization and in diagnosing gross defects such as destruction of turbine blades etc, causing a significant increase in the level of oscillations at certain frequencies. In the work of a real unit, the absolute repetition of the conditions of interaction of its parts with each other and with the external environment over time is almost impossible.

An adequate model of the process of oscillation excitation is the superposition of narrow-band random processes with multiple average frequencies:

$$
x(t)=\Sigma A_{k}(t) \supsetneqq o s \sharp\left[k \sharp \omega_{r} ¥-\varphi_{k}(t)\right]+\psi(t)
$$

in which $k \sharp \omega_{r}$ - average frequency of a narrow-band process; $A_{k}(t)$ - accidental, slow changeable envelope of the narrow-band process; $\varphi_{k}(t)$ - accidental, slow changeable phase; $\psi(t)$ - level of noise excitation.

The energy spectrum of this process is concentrated in the narrow frequency bands near $k f_{r}$.

When modeling oscillations excitation in rotary units, the change in the state of the unit can be estimated by change of not only in the magnitude of the amplitudes of the spectral components, but also in the level of noise excitation $\psi(t)$ with a uniform spectrum $S_{s h}(\omega) \mathrm{S}_{\mathrm{II}}$ in the given frequency range. This form of vibration signal representation simulates the wear of the contact surfaces of the teeth of the wheels in the gearbox, rolling bearings and the like quite well. 


\section{Analysis of recent references and publications}

Currently, two main areas of vibration monitoring of machinery and equipment are being actively developed. One of them solves the problem of minimizing the number of vibration control points and intervals between measurements, primarily through the use of stationary continuous monitoring systems. Within another, the intervals between measurements were maximized by controlling vibration at many points, in particular at each node of the equipment that is the source of vibration. As a result of increasing these intervals, it is possible to use portable equipment for measuring and analyzing vibration [4].

In both cases, effectiveness of monitoring depends on the number of vibration components available for measurement. To increase their number in machines with rotating nodes, a narrowband spectral analysis of vibration, the most effective for the analysis of periodic components of the signal, is done. As an example, Fig.1 shows the vibration spectra of the machine (reducer) without defects and after the appearance of defects in the gears, accompanied by an increase in the level (power) of many components of the vibration spectrum.
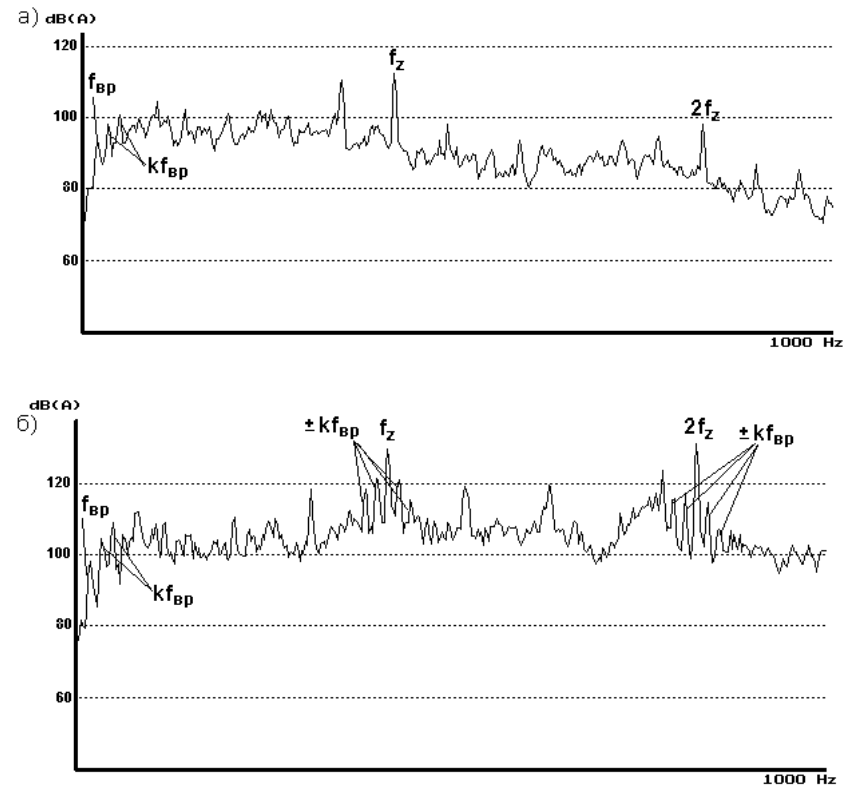

Fig. 1. V ibration spectra of reducer's bearing node: a - non-defect reducer; $\sigma$ - reducer with gear defect; fвp - reducer axis rotation speed; $f z$ - gear-meshing frequency.

M onitoring of the vibration state of the machine in this case lies in comparison of the level of individual components with the thresholds shown in Fig.2 and analysis of changes in the level of individual components during operation.

In practical diagnostics of vibration machines, there are two main approaches to solving diagnostic problems. In the first case, the diagnosis is performed only after detecting changes in the vibration state of the machine by means of monitoring and its task is to interpret these changes. However, monitoring is usually performed on low-frequency and medium-frequency vibration, which responds to the appearance of mainly developed defects only. They lead to noticeable changes in the energy parameters of vibration, which exceed their natural fluctuations when changing the operating modes of the machine [5]. 


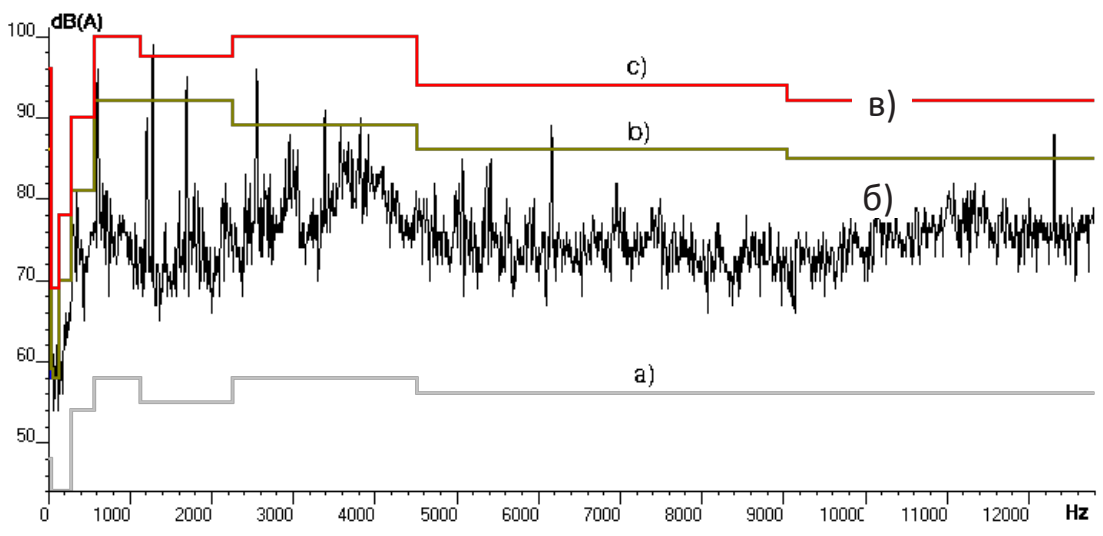

Fig. 2. M achines vibration state monitoring: a - weak vibration' signal threshold; $\sigma$ - average vibration' signal threshold; в - strong vibration' signal threshold.

The second approach is to use those methods and diagnostic tools that detect the main types of defects at the stage of their origin before there are significant energy changes in the vibration signal of the machine as a whole. Detection of defects at the stage of origin makes it possible to observe their development and timely plan work on repair and maintenance of the machine. This approach is often called defect monitoring, and it can be carried out for several years, until the combination of existing defects, both in depth and quantity, leads to a situation close to an emergency. Naturally, such an approach is possible only if all potentially dangerous defects can be detected and identified at an early stage of their development.

As a result of intensive development of diagnostics methods and technical means, and primarily on a vibration signal, present-day systems of defects monitoring become a reality and become more and more widespread.

To detect emerging defects in rotating nodes, natural diagnostic features are used, which are determined by spectral analysis of the vibration signal itself or the power fluctuations of its components (spectral analysis of the envelope).

To form a diagnostic feature, the signal is represented in a rather narrow frequency range, for example, in the area of one of the harmonics of the fundamental excitation frequency of the mechanism (gear, screw, blade, etc.).

A the same time, the oscillations are represented in the form of modulation of the highfrequency harmonic signal by the sum of the harmonic low-frequency oscillations.

Figure 3 shows a narrowband random process (amplitude modulation), which is described by the following mathematical expression:

$$
y(t)=A[1+m E(t)] \cos \left(\omega_{0} t+\varphi_{0}\right),
$$

in which $\omega_{0}$ - carrier frequency, for instance, gear tooth frequency; $A$ - apmlitude; $m$ modulation depth (varies from 0 to 1 ); $E(t)$ - overall sum of harmoniuos low-frequency oscillations multiple of basic excitation frequency $\Omega_{0}$, for instaance, gear rotation frequency;

$$
E(t)=\Sigma B_{k} \cos \left(k \Omega_{0} t+\varphi_{k}\right)
$$

Isolation of the envelope is done using an amplitude detector [6]. 


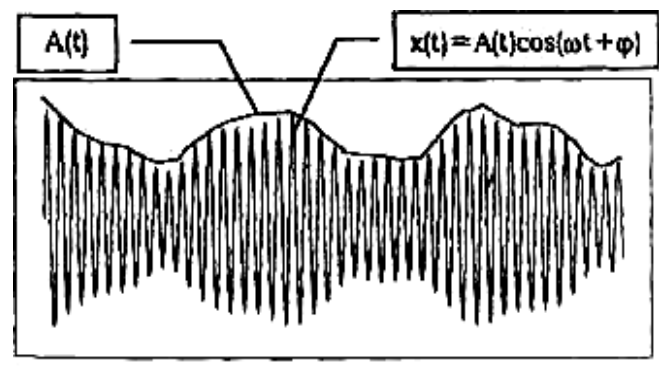

a)

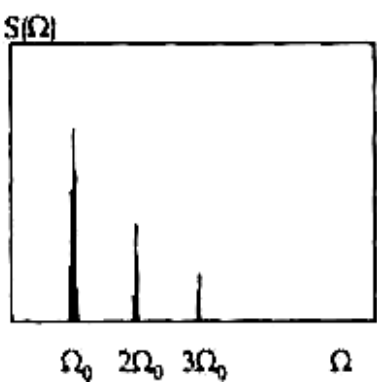

b)

Fig. 3. Narrow-band accidental process (solid line) and its envelope (dotted line) (a) and envelope spectrum (б).

To process such signals, the integrated programming environment made in style of $M$ icrosoft $V$ isual-products has been developed (fig. 4). A working project can have three types of modules: system, drivers and programs. The system module determines hardware configuration and external devices (tools) with the appropriate drivers used. The software module contains one or more programs that are built in the style of tests and tasks. A task is a group of tests. A typical test is the procedure of measuring and issuing its result. The result of the test can also be a response to the compliance of the obtained data with the specified requirements. The test results are recorded in the protocol.

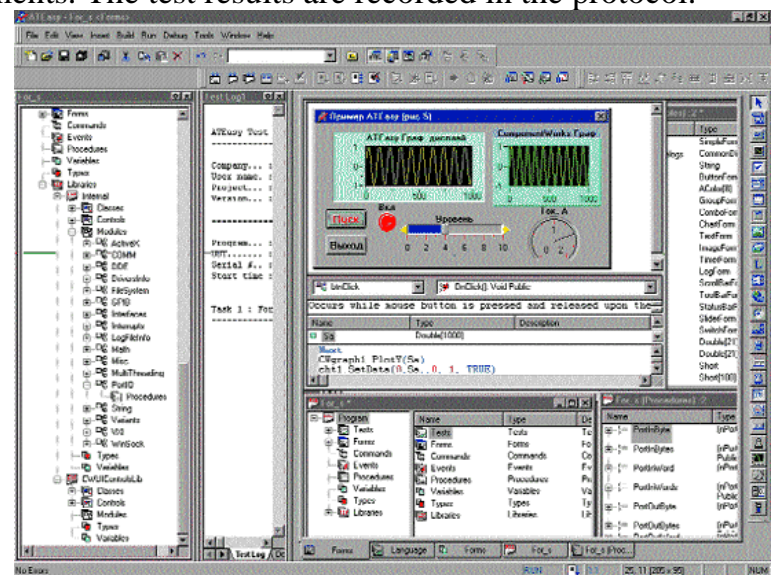

Fig. 4. A TEasy programming environment.

Each module includes sub-modules necessary for their functioning in the form of libraries, commands, variables, events, etc. The built-in system library (Internal) is based on Microsoft COM (Component Object Model) technology and provides tools for creating dialog boxes.

External libraries that contain COM objects and A ctivex components can be used. In the example in Figure 2, for the purpose of demonstration, a library of A ctivex components was used for user interface with Component Works when creating another graphical display. This structure of the package significantly expands its capabilities and scope.

Figure 5 shows the display panels of the oscilloscope (Time Series), spectrum analyzer (Spectrum), the graph of the change of the spectrum in time, in which instant "snapshots" of the signal spectrum are drawn vertically with colored lines (Spectrogram), threedimensional spectrogram (3-D Surface) and the control panel of the combined device 
("Run", "Stop", buttons etc.). The sizes and mutual arrangement of graphic panels are adjusted arbitrarily, at the request of the operator.

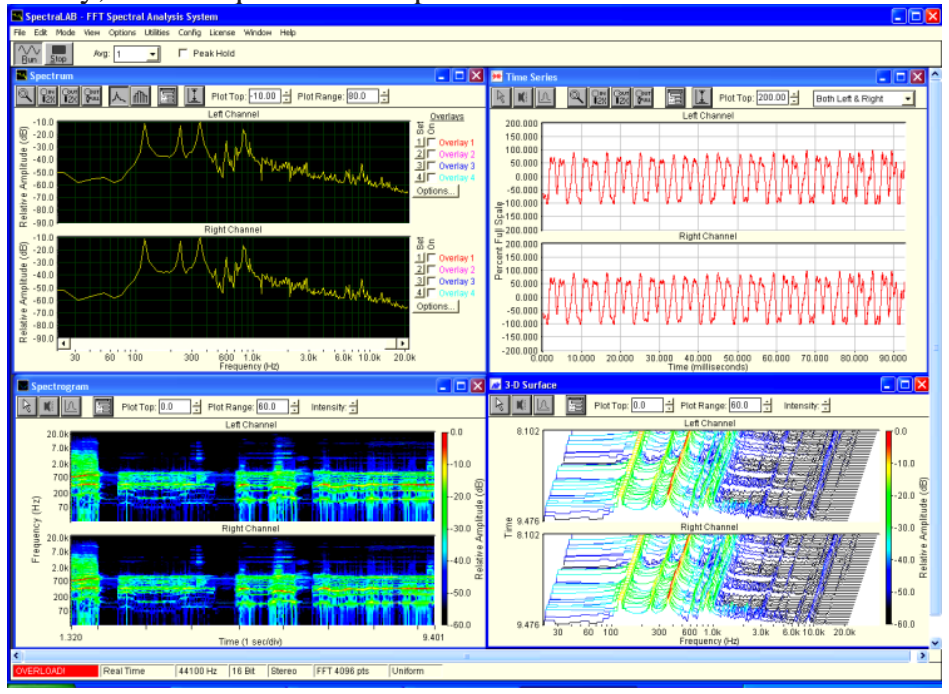

Fig. 5. Indication panels of Spectrum $L$ ab software.

In order to perform vibration diagnostics of sewing equipment, the following method of spectral analysis is proposed.

To obtain the maximum value of the signal-to-noise ratio and the optimal selection of useful components from the vibration signal, it is proposed to use a modulated signal in digital form as a reference (modulating) signal. A nalysis of existing audio file processing software has made it possible to improve and offer to use Spectrum Laboratory software product.

A specific feature of Spectrum Lab software is another menu item - Spectrum $L a b$ Components (Fig. 6), where in the form of an equivalent block diagram, the "iron" equivalent of software blocks is shown, highlighting involved in a particular mode nodes of the scheme with green color, gray - "disabled" and red - blocks whose parameters are set incorrectly or modes do not meet the maximum permissible (for instance, overloaded sound card mixer).

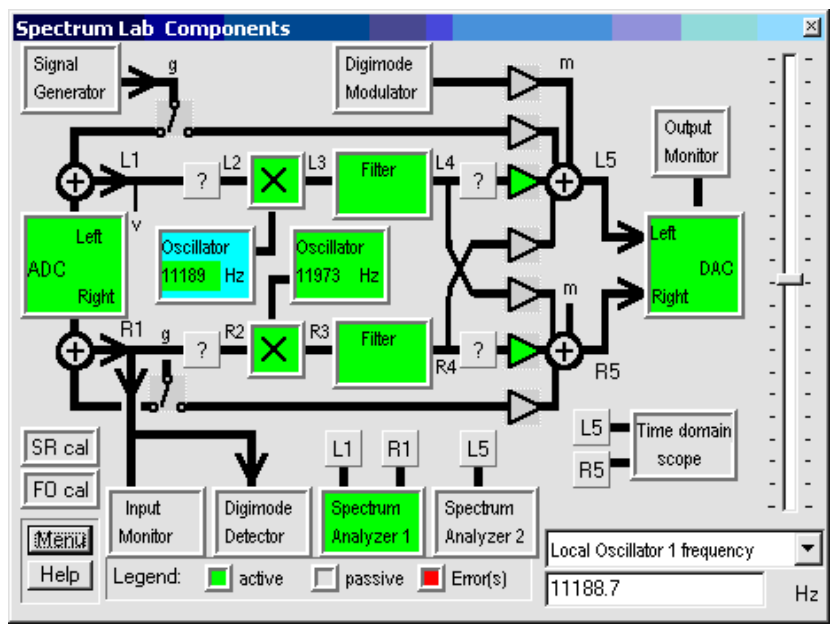

Fig. 6. Spectrum L ab Components indication window. 


\section{Presenting main material}

To identify the characteristic diagnostic features, the vibroacoustic process is subjected to preliminary spectral processing, which allows to identify the zones and the nature of the largest changes in the signal in the frequency domain. B ased on spectral analysis, a method is selected to separate the informative component from the signal by suppressing interference through filtering, integration, detection, averaging, and so on. Then a list (dictionary) of diagnostic signs sensitive to a certain defect is created $[3,6]$.

We assume that the vibration signal of the working unit contains all the information about the interaction of its components and parts. The task of vibromonitoring is to develop algorithms for obtaining information about the state of a particular node and the localization of faults that occur [7].

Technical means of measuring and analyzing signals in stationary systems for monitoring and diagnostics of machines are not structurally and functionally different from the means used in portable systems $[8,9]$. The only difference is in the technical implementation, which is related to the need to repeat the measurements at the same control points with such a short time interval, which would ensure the timely shutdown of the machine even in the event of avalanche-like defects. A typical structure of a stationary system is shown in Fig. 7.

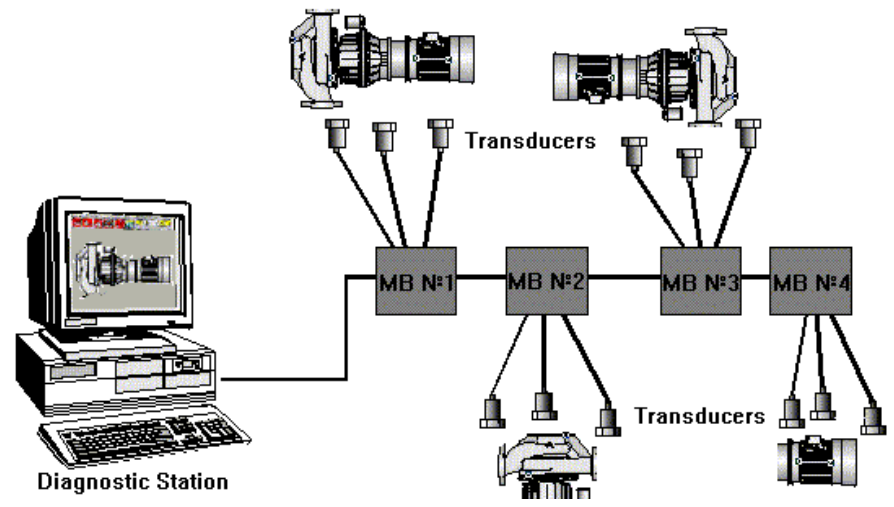

Fig. 7. Structure of monitoring and diagnostics stationary system.

The number of units for measuring and analyzing signals in stationary systems is usually determined by the number of control points and the maximum permissible interval between measurements. The number of sensors per unit can range from one to several dozen. The function of the measurement unit includes the analysis of vibration or noise, as well as other physical quantities according to the program set by the diagnostic center. The algorithms of the program automatically change depending on the diagnostic results, i.e. the state of the object of control. Sometimes the function of the measurement unit is to compare the results of measurement and analysis with the thresholds that set the limits of the permissible change in diagnostic parameters.

If the permissible time between periodic measurements is large enough, the system can use one unit, to which the sensors are connected via electronic signal switching devices. The measuring unit can be structurally integrated into one case with the diagnostic center. The diagnostic center is either a single computer connected to a single network with measurement units, or a group of computers running in parallel or with a separation of functions.

B ased on the above-mentioned, to ensure the requirements of optimal vibration analysis, the structure of the control system proposed by the state of the equipment has the form shown in Fig.8. 


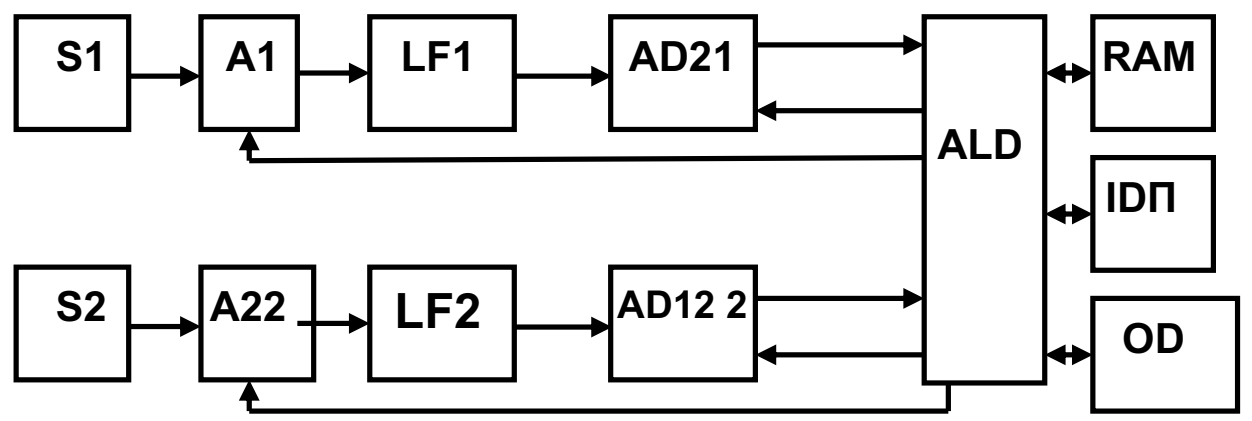

Fig. 8. Structural scheme of the system of sewing equipment state control: S1, S2 - vibration sensors; A 1, A 2 - amplifiers with variable amplification coefficient; LF1, LF 2 - lower frequencies band filters; AD 1, AD 2 - analog and digital converters; ALD - arithmetically logical device; RA M random access memory; ID - input device (mouse, keyboard); OD - output device (display).

The analysis of random vibration of the diagnosed object should be done using real-time two-channel analyzers. A processor is installed in each channel of the analyzer for fast Fourier transform and fast information processing. The two available channels provide an opportunity to assess the state of the object by spectral-correlation functions, as well as by cepstrum. The results of the analysis are displayed.

For practical implementation of the proposed system of automated control and diagnosis of technical condition of light industry equipment it is necessary to provide recommendations for practical selection of its elements, to determine the features of setting control points for vibration and establish the procedure to determine the nature of the defect $[13,14]$.

The sensor should be fixed so that its measuring axis coincides with the direction required for measurement. As a rule, the measuring axis is perpendicular to the plane of fastening of the sensor (its working surface). The purpose of measurement and analysis of vibration usually defines the location of the attachment of the piezoelectric sensor on the object being researched (Fig. 9).

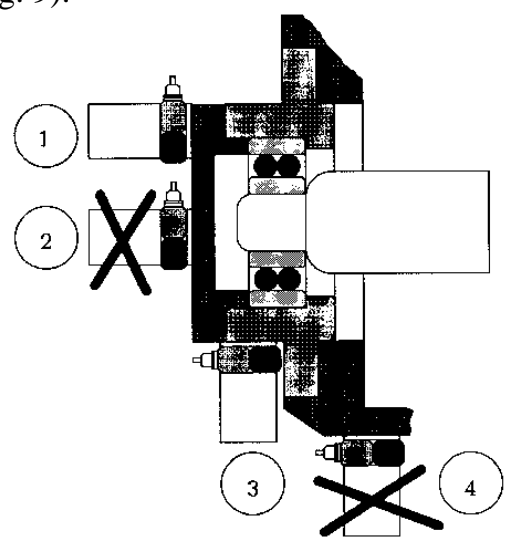

Fig. 9. Recommended spots to install the sensor.

In most cases, the purpose of vibration measurements is to monitor the operating conditions of the shaft and bearing. The sensor should be installed so that mechanical vibrations of the bearing act directly on its working surface $[15,16,17]$. The sensor 3 perceives the mechanical oscillations of the bearing with less vibration, which is disturbed by other components and parts of the unit, compared to sensor 4, which receives the transformed bearing oscillations and mechanical oscillations when passing through the 
detachable connection, generated by other units. Similarly, the sensor 1 is placed more appropriately in terms of distribution of mechanical vibrations than the sensor 2 . $M$ easurement of vibration in the thin sections of cases and covers is unacceptable [10].

\section{Results of experimental studies}

To conduct research and determine the specific values of the spectral components frequencies for a particular sample, an experimental installation shown in Fig.10 was developed. The main elements of the experimental installation are: sewing machine 1; sensors to measure vibration 2; signal matching unit 3; analog-digital device 4, computer 5.

The measuring equipment works on the principle of analog-to-digital conversion of a current signal, by means of high-precision vibration sensors and analog-digital device with the subsequent output of experimental data on the screen of the computer [11].

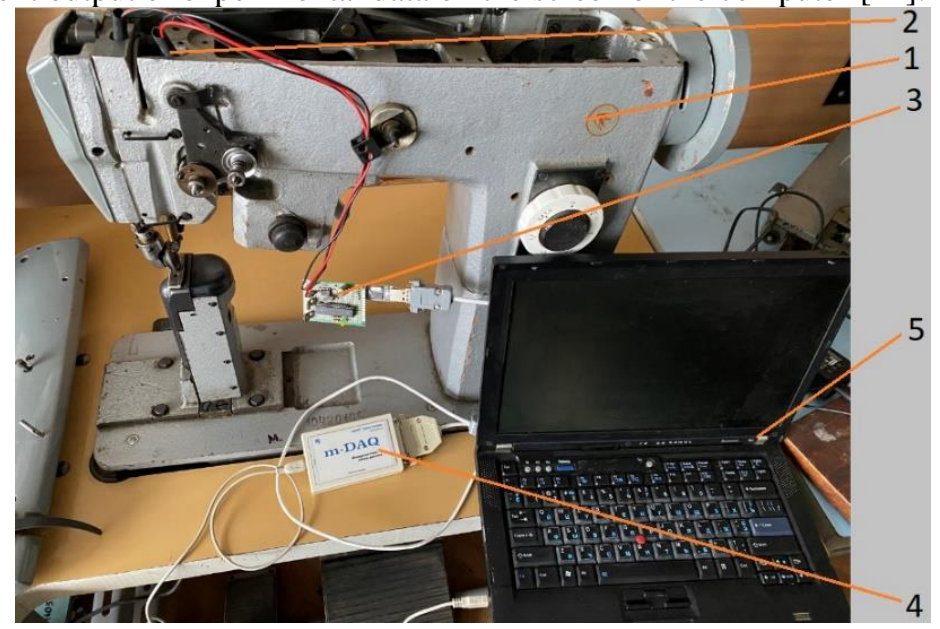

Fig. 10. Experimental installation for sewing machine diagnostics.

In the detailed diagnostics mode, we can obtain vibration spectra of the node and the spectrum of its high-frequency envelope components, which makes it possible to identify components and indicate the possible affiliation of the identified components to a certain sign of the defect. As a result of research of connection of signal values with the spectrum, some recommendations on a choice of these parameters are given.

For instance, we have a recorded time signal of vibration speed with the following parameters: number of samples $\mathrm{n}=512$; time between counts (step) $\Delta \mathrm{t}=0,1 \mathrm{~ms}$; measurement time $T=51.1 \mathrm{~ms}$. Using the obtained values according to the appropriate formulas, the parameters of the spectrum were calculated: the number of lines in the spectrum $\mathrm{N}=257$, the resolution of the spectrum $\Delta \mathrm{F}=19569 \mathrm{~Hz}$, the upper frequency of the spectrum $\mathrm{F}=5010 \mathrm{~Hz}$.

For accurate diagnostics on a spectrum it is necessary to choose the number of samples (4096 or 8192). With a larger number of samples, errors occur with the precise determination of the amplitude and, especially, the phase of the harmonic: it breaks into several components.

The calculations show that in order to obtain a range of low-speed machines, the parameters need to be taken for a long period of time. Thus, to get a harmonic of $0.1 \mathrm{~Hz}$ (this is 6 rpm) parameters must be taken for at least 10 seconds.

The upper frequency of the spectrum is usually chosen so as to obtain 10-20 harmonics from the frequency value. For instance, for $50 \mathrm{~Hz}(3000 \mathrm{rpm})$ - it is $500-1000 \mathrm{~Hz}$. In some cases of diagnostics, more high-frequency harmonics of $1000 \mathrm{~Hz}$ and above, but no more 
than $5000 \mathrm{~Hz}$ are required. In other case there will arise problems with sensors and devices, and it will be difficult to receive a normal spectrum.

To facilitate the diagnosis of equipment calculations, signal processing parameters are summarized in table 1.

Table 1. Parameters of signal processing for various rotation frequencies.

\begin{tabular}{|c|c|c|c|}
\hline $\begin{array}{c}\text { Rotation frequency, } \\
\mathrm{Hz} \text { (rot/min) }\end{array}$ & $\begin{array}{c}\text { Intervals between } \\
\text { counts in the signal to } \\
\text { obtain 1600 lines, ms }\end{array}$ & $\begin{array}{c}\text { Intervals between } \\
\text { counts in the signal to } \\
\text { obtain 3200 lines, ms }\end{array}$ & $\begin{array}{c}\text { A pproximated time of } \\
\text { measurement, s }\end{array}$ \\
\hline $1(60)$ & 2 & 1 & 8 \\
\hline $5(300)$ & 0,5 & 0,25 & 2 \\
\hline $10(600)$ & 0,2 & 0,1 & 0,8 \\
\hline $25(1500)$ & 0,1 & 0,05 & 0,4 \\
\hline $50(3000)$ & 0,05 & 0,025 & 0,2 \\
\hline $100(6000)$ & 0,02 & 0,01 & 0,08 \\
\hline
\end{tabular}

When analyzing the spectra of accumulated measurements, either an increase in their amplitudes during observations, or an increase in their amplitudes above the average value, which is automatically calculated by a group of vibration measurements of identical nodes of several identical machines are indicated in the table of detected components.

Figure 11 shows the spectral components of the experimental sample (sewing machine). The selected value of the spectral component $(87.3537 \mathrm{~Hz})$ is close to the calculated parameters of the gear-belt transmission between the main and distribution pulleys of the machine. The relative level of vibration ( $75-55=20 \mathrm{~dB})$ indicates its increase and needs to be eliminated.

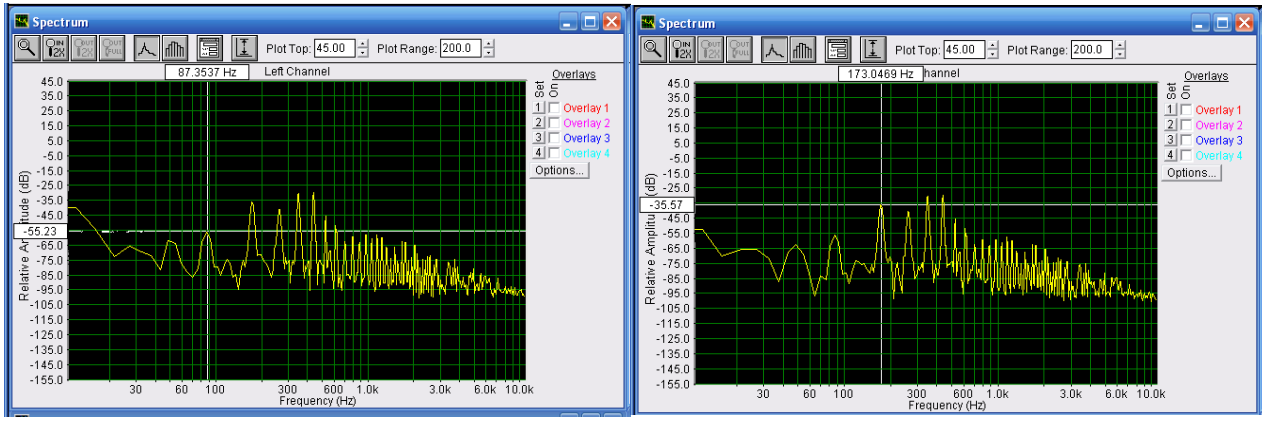

a)

б)

Fig. 11. Spectral component of distribution pulley (a) and shuttle pulley (б) vibration.

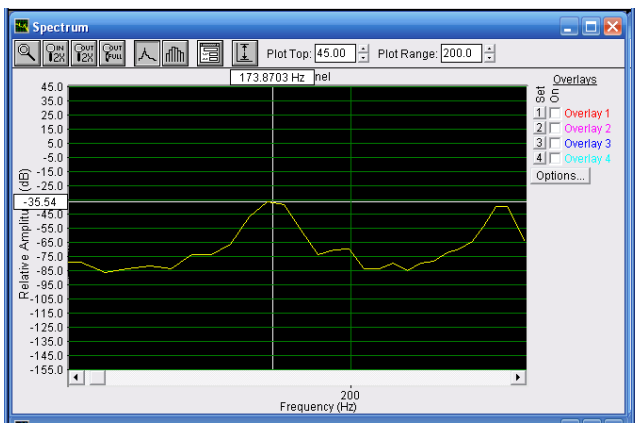

a)

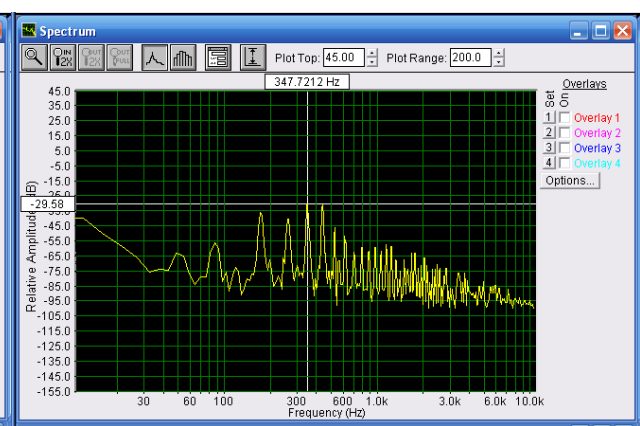

b)

Fig. 12. Spectral component (2nd harmonic) of distribution pulley (a) and shuttle pulley (б) vibration. 
The results of the study presented in Fig.12 on the value of the spectral component $(173.0469 \mathrm{~Hz})$ indicate an increased level of vibration of the driven pulley of the shuttle mechanism, while clarification of the frequency with increasing resolution proves it.

Moreover, the measurements indicate available vibration harmonics of the shuttle mechanism, which confirms the diagnosis.

Thus, the use of the developed method of diagnostics of light industry equipment, namely the sewing machine, made it possible to determine its faulty unit.

\section{Conclusions}

As a result of this research, a new technique and algorithm for obtaining and processing vibrating signals have been developed. Their difference from the previously known ones is determined by the use of a newly created vibration sensor (electronic stethoscope) on the basis of a piezoceramic microphone. The main difference in the processing of vibration signals lies in obtaining a mutually correlated function from the two signals received on the equipment case and at the point of node diagnosis defined by the kinematic scheme. Also, the created methods of determining the frequency range of defects of different units of light industry equipment differ from those known by taking into account the size of the parts of the equipment units and the possibility of narrowband filtering. Prospects for the development of stationary monitoring systems are also associated with the development of microcomputers. The result of this development may be separation of functions between the measurement units and the diagnostic center. The measuring unit can take over the monitoring functions by contacting the diagnostic center only in the event of defects in order to identify them. Obviously, in this case, one diagnostic system can work with a large number of measurement units, monitoring the condition of the equipment of the entire enterprise.

\section{References}

1. B. Watts, J. Van Dyke, An automated vibration - based expert diagnostic system, Sound and vibration, September, (1993)

2. F. J. Harris, On the Use Windows for Harmonic Analysis with the Discrete Fourier Transformation, Proceedings of the IEEE, vol. 66, p. 51-83, (1978)

3. A. D. Poularikas, The Handbook of Formulas and Tables for Signal Processing, CRC Press LLC, (1999)

4. A. Muszynska, Vibrational Diagnostics of Rotating Machinery Malfunctions, International J ournal of Rotating M achinery - 1, p. 3-4, (1995)

5. A. V. Barkov, N. A. Barkova, A. Y u Azovtsev, M onitoring and diagnostics of rotor machines by vibration, St. Petersburg, СПбГМТУ Publishers, p. 160, (2000)

6. J. Musiał, S. Horiashchenko, R. Polasik, T. Kałaczyński, M. M atuszewski, M. Śrutek, Abrasion Wear Resistance of Polymer Constructional Materials for Rapid Prototyping and Tool-Making Industry, Polymers, 12, 873, (2020)

7. D. A bboud. Vibration based condition monitoring of rotating machines in nonstationary regime, M echanics, INSA deL yon, (2015)

8. S. Horiashchenko, I. Golinka, A. B ubulis, V. J urenas, Simulation and Research of the Nozzle with an Ultrasonic Resonator for Spraying Polymeric Materials, Mechanika, vol. 24, no 1, p. 61-64, (2018)

9. S. Horiashchenko, K. Horiashchenko, J. Musiał, Methodology of measuring spraying the droplet flow of polymers from nozzle, M echanika, 26, p. 82-86, (2020)

10. K. J. Sinha, Significance of Vibration Diagnosis of Rotating Machines during Installation and Commissioning: A Summary of Few Cases, Noise\& Vibration W orldwide, (2006)

11. D. Yakymchuk, O. Yakymchuk, O. Chepeliuk, N. M yrhorodska, J. Koshevko, 0. 
Orlenko, I. Nosova, Study of cutting presses in designing a women's costume for hospitality industry, Eastern-European Journal of Enterprise Technologies, Engineering technological system, vol. 5, no. 1 (89), p. 26-36, (2017)

12. O. Yakymchuk, D. Yakymchuk, N. Kushevskiy, E. Chepelyuk, J. Koshevko, N. M yrhorodska, O. Dzyundzya, V. Burak, Prerequisites for the development of hydrojet technology in designing women's headgear at hospitality establishments, EasternEuropean J ournal of Enterprise Technologies, Engineering technological system, vol. 1 , no. 1 (91), p. 36-46, (2018)

13. A. Dykha, S. Matyukh, T. Kałaczyński, Diagnostics - Experimental A nalysis of Friction Pairs at Stick - Slip Sliding, 18th International Conference Diagnostics of M achines and V ehicles, M A TE C W eb of Conferences, 302, (2019)

14. M. Łukasiewicz, M. Liss, N. Dluhunovych, Analysis of vibrodiagnostics methods in the technical state study of designed multimedia mobile scenes, 18th International Conference Diagnostics of Machines and Vehicles, MATEC W eb of Conferences, Vol. 302 (2019)

15. V. M artynyuk, M. Liss, J. Wilczarska, Diagnostics of Corrosion Protection Potential for Electric W ater Heaters, 18th International Conference Diagnostics of M achines and V ehicles, M A TEC W eb of Conferences, V ol. 302 (2019)

16. M. Żółtowski, M. Liss, J. Melcer, Vibration diagnostics of concrete block, 17th International Conference Diagnostics of Machines and Vehicles, MATEC Web of Conferences, Vol. 182 (2018)

17. I. Kovtun, J. Boiko, S. Petrashchuk, T. Kałaczyński, Methods for Vibration Reduction in Enclosed Electronic Packages, 18th International Conference Diagnostics of M achines and V ehicles, M ATEC W eb of Conferences, 302, (2019) 